\title{
The clinical development of obinutuzumab for the treatment of follicular lymphoma
}

This article was published in the following Dove Press journal:

Cancer Management and Research

10 April 2017

Number of times this article has been viewed

\author{
Barbara $\mathrm{Ma}^{\prime}$ \\ Chaitra Ujjani² \\ 'Department of Medicine, ${ }^{2}$ Lombardi \\ Comprehensive Cancer Center, \\ MedStar Georgetown University \\ Hospital, Washington, DC, USA
}

\begin{abstract}
Impressive progress has been made in recent decades for advanced-stage follicular lymphoma with the availability of anti-CD20 monoclonal antibodies, initially rituximab and more recently obinutuzumab. Obinutuzumab is a unique, third-generation, fully humanized glycoengineered $\mathrm{IgG}_{1}$ type II anti-CD20 monoclonal antibody. It has been shown to have increased antitumor activity compared to rituximab in preclinical studies, including wholeblood B-cell depletion assays, xenograft models, and primate models. This has spurred on the development of obinutuzumab through Phase I/II trials as monotherapy and in combination with chemotherapeutic agents and other targeted therapies. Its efficacy compared to rituximab and in rituximab-refractory disease has led to its continued development and eventual approval for the treatment of follicular lymphoma. Here in this review, we highlight the design and development of obinutuzumab in the treatment of advanced stage grade 1-3A follicular lymphoma and its future directions.
\end{abstract}

Keywords: follicular lymphoma, obinutuzumab, anti-CD20 monoclonal antibody

\section{Introduction}

Follicular lymphoma (FL) is the second most common subtype of non-Hodgkin's lymphoma (NHL), accounting for $35 \%-40 \%$ of all adult lymphomas in the US. ${ }^{1} \mathrm{FL}$ is usually clinically characterized as an indolent disease, though its course is highly variable. The World Health Organization (WHO) classification system for FL is based on the relative proportion of centrocytes to centroblasts, with a greater proportion of centroblasts more likely to behave aggressively. ${ }^{2}$ While grades $1-3 \mathrm{~A}$ have an indolent clinical course, increasing evidence suggests that grade $3 \mathrm{~B}$ is a biologically distinct entity that histologically resembles diffuse large B-cell lymphoma (DLBCL) and is clinically more aggressive. Because of the high radiosensitivity of FL and the potential for cure at early stages, radiotherapy is sometimes recommended for limited-stage FL patients. For patients without symptoms and low tumor burden, patients may opt for a "watch-and-wait" approach, due to the indolent course of FL. Many patients remain asymptomatic despite extensive disease, with the vast majority of patients diagnosed at advanced stages. However, FL is considered incurable despite standard therapies, and patients with advanced FL often suffer from disease relapse or progression of disease. Impressive progress has been made in recent decades in the treatment of advanced-stage FL with the availability of anti-CD20 monoclonal antibodies, including the chimeric rituximab and more recently the humanized obinutuzumab.
Correspondence: Chaitra Ujjani MedStar Georgetown University Hospital, 3800 Reservoir Road Northwest, Washington, DC 20007, USA

Tel +l 2024440596

Fax + I 2024449429

Email csu@georgetown.edu 


\section{Anti-CD20 monoclonal antibodies}

Anti-CD20 monoclonal antibodies can be classified as type I and type II (Table 1). Type I antibodies mediate the translocation of CD20 into lipid rafts and recruit C1q of the complement cascade to induce complement-dependent cytotoxicity potently, as well as antibody-dependent cellmediated cytotoxicity, though they are relatively poor at inducing direct cell death. ${ }^{3,4}$ In contrast, type II antibodies have a lower level of complement-dependent cytotoxicity (CDC) in vitro, but instead promote strong homotypic adhesion and have a strong induction of direct cell death via non-caspase-dependent pathways.

\section{Rituximab}

Rituximab is a chimeric type I CD20 monoclonal antibody $(\mathrm{mAb})$ that structurally consists of a human $\mathrm{K}$-constant region, a human $\operatorname{IgG~Fc}$ portion $\left(\mathrm{IgG}_{1}\right)$, and murine variable region that recognizes the human $\mathrm{CD} 20$ protein. ${ }^{5}$ As rituximab is a type I $\mathrm{mAb}$, signaling induced by it involves raft microdomains and causes activation or inhibition of several pathways responsible for apoptosis, proliferation, and survival. It primarily works through three mechanisms of action to eliminate CD20-positive cells: 1) induction of apoptosis, 2) CDC, and (3) antibody-dependent cellular cytotoxicity mediated through Fc $\gamma$ receptor-expressing cells, such as natural killer (NK) cells, $\gamma \delta$ T lymphocytes, and macrophages. Rituximabbased chemoimmunotherapy has become the standard of care for frontline treatment of advanced-stage FL, based on several major prospective randomized studies that uniformly demonstrated a significant increase in overall response rate (ORR), progression-free survival (PFS), and particularly overall survival (OS) compared to chemotherapy alone. ${ }^{6-10}$ More recent studies, including the STIL NHL1, BRIGHT,

Table I Features of Type I and II monoclonal antibodies

\begin{tabular}{|c|c|}
\hline & \\
\hline $\begin{array}{l}\text { Intertetramer binding } \\
\text { - Localization of CD20 onto lipid } \\
\text { rafts } \\
\text { - High complement-dependent } \\
\text { cytotoxicity induction } \\
\text { - Binding to class I epitope } \\
\text { - No homotypic adhesion } \\
\text { - Caspase-dependent induction of } \\
\text { cell death by apoptosis } \\
\text { - Full CD20-binding capacity } \\
\text { - CD20 modulation } \\
\text { - Examples: rituximab, } \\
\text { ofatumumab }\end{array}$ & $\begin{array}{l}\text { Intratetramer binding } \\
\text { - No localization of CD20 onto } \\
\text { lipid rafts } \\
\text { - Low complement-dependent } \\
\text { cytotoxicity induction } \\
\text { - Binding to class II epitope } \\
\text { - Strong homotypic adhesion } \\
\text { - Inducing non-caspase-dependent } \\
\text { - direct cell death } \\
\text { - Half-maximal CD20 binding at } \\
\text { - Lesturating conditions } \\
\text { - Examples: obinutuzumab, } \\
\text { tositumomab }\end{array}$ \\
\hline
\end{tabular}

and FOLL05 trials, have provided guidance regarding the chemotherapy backbone in regard to toxicity and PFS, and continue to utilize rituximab. ${ }^{11-13}$

Malignant B cells can become resistant to rituximab after prior successful treatment. Several mechanisms have been proposed for rituximab resistance, including low-

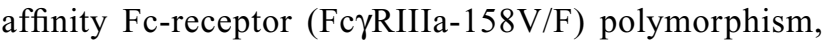
overexpression of complement-inhibitory molecules CD55 and CD59, high tumor burden, and low level of CD20 expression. ${ }^{14,15}$ Czuczman et al also showed that repeated exposure to rituximab can lead to acquired downregulation of CD20 from reduced messenger RNA levels and posttranscriptional modifications. ${ }^{16}$ Resistance occurs in about half of the patients, leading to early relapse or refractory disease. Patients whose disease fails to respond to a rituximab-containing regimen have few treatment options and a poor prognosis. Therefore, better treatment options are needed.

\section{Development of second- and third- generation CD20 mAbs}

Several second- and third-generation murine, humanized, ${ }^{17}$ or completely human mAbs targeting CD20 have been developed. ${ }^{3}$ Ofatumumab, which is a fully human $\mathrm{IgG}_{1} \kappa$ type $\mathrm{I}$ anti-CD20 mAb, was the first to be approved by the US Food and Drug Administration (FDA), specifically for relapsed chronic lymphocytic leukemia (CLL) after fludarabine and alemtuzumab. ${ }^{17}$ Compared with rituximab, ofatumumab binds a unique seven-mer loop of the human CD20 molecule that is in closer proximity to the cell membrane than the binding site of rituximab, which binds a 44-mer loop. ${ }^{18}$ This binding of ofatumumab to this location enables the ability to bind and activate more $\mathrm{Clq}$ at the cell surface and mediate enhanced CDC. In a Phase I/II study of ofatumumab in relapsed or refractory FL patients, ofatumumab demonstrated high single-agent activity and achieved an ORR of $43 \%$ and median time to progression of 8.8 months. ${ }^{19}$ However, it demonstrated minimal activity in rituximab-refractory FL patients, with a modest ORR of $11 \%$ and median PFS of 5.8 months. ${ }^{20}$

Obinutuzumab (GA101; Gazyva/Gazyvaro) is a novel glycoengineered type II anti-CD20 antibody that induces enhanced antibody-dependent cell cytotoxicity and direct cell death compared with rituximab. It has proven effective in both relapsed and rituximab-refractory FL, as well as previously untreated patients. This review focuses on obinutuzumab in the treatment of advanced-stage WHO grade 1-3A FL. 


\section{Obinutuzumab}

\section{Structural characteristics and mechanism of action}

Obinutuzumab is a unique, third-generation, fully humanized, glycoengineered $\mathrm{IgG}_{1}$ type II anti-CD20 mAb that was designed to enhance B-cell killing compared to rituximab. It recognizes a CD20 epitope overlapping with that of rituximab, but it exhibits a different elbow-hinge angle and binds CD20 in a different orientation compared with type I anti-CD20 antibodies. ${ }^{21}$ The new spatial arrangement between $\mathrm{CD} 20$ and obinutuzumab allows obinutuzumab to bind its two Fab arms on the same CD20 tetramer, which rituximab is structurally unable to do. The intra-CD20tetramer binding for type II antibodies (ie, obinutuzumab) compared to inter-CD20-tetramer binding for type I mAbs (ie, rituximab) led to the proposed dynamic model of how this type of binding is felt to increase antibody-dependent cellular cytotoxicity (see Table 1). ${ }^{22}$ As expected for a type II $\mathrm{mAb}$, obinutuzumab supplements B-cell killing with nonapoptotic cell-death mechanisms via homotypic adhesion, lysosomal cathepsin release, ${ }^{23}$ and production of radical oxygen species. ${ }^{24}$ In addition, the Fc portion of obinutuzumab has been glycoengineered to reduce fucosylation, result-

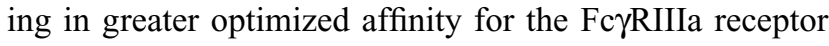
and translating into enhanced antibody-dependent cellular cytotoxicity potency, despite lower CDC activity compared to rituximab. ${ }^{25,26}$ The increase in affinity between afucosylated antibodies and FcyRIIIa has been shown to enhance activation of signaling molecules, promoting cytoskeletal rearrangement and degranulation, and subsequently enhance the cytotoxic characteristics of NK cells to increase efficiency of antibody-dependent cellular cytotoxicity. ${ }^{27}$ These mechanisms of obinutuzumab as a type II antibody may provide an advantage when compared with rituximab, in order to achieve increased clinical activity.

\section{Preclinical studies}

In preclinical studies, obinutuzumab was shown to have increased antitumor activity compared to rituximab. In a head-to-head comparison of obinutuzumab, rituximab, and ofatumumab, clinically relevant doses of obinutuzumab demonstrated good efficiency in controlling disease progression of an aggressive DLBCL model (SUDHL4 cells) and the ability to induce complete remission, while identical doses of rituximab and ofatumumab could not. ${ }^{28}$ This was also noted in rituximab-refractory FL tumor models, ${ }^{29}$ as well as 3-D multicellular aggregates of lymphoma-cell models. ${ }^{30}$ Other xenograft model studies have suggested that the combination of obinutuzumab and chemotherapy, such as chlorambucil, fludarabine, and bendamustine, or cyclophosphamide was more effective than combinations of rituximab with chemotherapy in terms of antitumor efficacy. ${ }^{29,31}$ In primates, obinutuzumab and rituximab induced similar peripheral blood B-cell depletion, though obinutuzumab was able to achieve superior B-cell depletion compared to rituximab in deep lymphoid tissue and splenic tissue. ${ }^{26}$ The decreased ability of obinutuzumab to fix complement relative to rituximab results in an enhanced ability of obinutuzumab to bind to NK cells, activate NK cells, and induce antibody-dependent cellular cytotoxicity more effectively than rituximab in the presence of complement. ${ }^{32}$

\section{Pharmacokinetics}

Pharmacokinetics for identifying optimal obinutuzumab dosing and schedule were derived from indolent NHL and aggressive NHL patients in the Phase I/II GAUGUIN and Phase IB GAUDI studies. ${ }^{33}$ Given the differences in mechanism of action, nature of CD20 binding, and signaling pathways activated, the obinutuzumab-dosing schedule could not be extrapolated from rituximab. In GAUGUIN, patients received up to nine infusions of obinutuzumab monotherapy at doses of 50-2,000 mg. Pharmacokinetic data obtained during Phase I dose escalation showed that serum obinutuzumab concentrations after doses of 400/800 mg yielded substantial increases in serum obinutuzumab concentrations compared with the lower doses. ${ }^{34}$ Additionally, there was a tendency for serum concentrations to increase over the treatment course.

Therefore, two doses of obinutuzumab were selected for evaluation in GAUGUIN Phase II: a low-dose cohort receiving 400/400 $\mathrm{mg}$ and a high-dose cohort of 1,600/800 $\mathrm{mg}$. The higher dose arm was identified as likely to produce the highest serum concentrations of obinutuzumab in NHL patients, as demonstrated by the stronger response (55\%) in indolent NHL patients compared to the lower dose arm $(17 \%) .{ }^{33}$ There were also more marked decreases in tumor size at higher doses and higher obinutuzumab concentrations. Weight and sex did have influence on pharmacokinetics, with increased exposure of obinutuzumab in women or in individuals with lower body weight in the $400 / 400 \mathrm{mg}$ group. These differences in obinutuzumab serum concentration with sex and weight were not observed in the $1,600 / 800 \mathrm{mg}$ group. Therefore, the $1,600 / 800 \mathrm{mg}$ dose was found to be the likeliest to produce the highest serum concentrations of obinutuzumab in NHL patients. The Phase II dose selection was based on modeling and simulation of pharmacokinetic data, which showed faster elimination of obinutuzumab in 
the first cycle than in later cycles, indicating a need for a more dose-dense regimen in the first cycle. However, a single dose for all infusions was thought to be more desirable, due to convenience and ease of administration compared to a size-adjusted dose. ${ }^{33}$ Therefore, the regimen of obinutuzumab $1,000 \mathrm{mg}$ administered on day 1 (D1) and D8, with an additional infusion on D15 of a 21-day cycle (C1) was proposed. Pharmacokinetic simulation showed that this regimen would result in trough serum concentrations of obinutuzumab similar to those observed with the $1,600 / 800 \mathrm{mg}$ dose. Therefore, the fixed dose of obinutuzumab 1,000 mg on D1, D8, and D15 of C1 and D1 of C2-C8 was selected for subsequent clinical trial assessment in patients with CD20-positive NHL.

\section{Development Obinutuzumab monotherapy trials} Phase I

In the initial multicenter Phase I GAUGUIN study, 21 heavily pretreated patients with relapsed or refractory $\mathrm{CD} 20^{+}$ indolent NHL received obinutuzumab monotherapy in a doseescalating fashion of 50/100 mg, 100/200 mg, 200/400 mg, $400 / 800 \mathrm{mg}, 800 / 1,200 \mathrm{mg}, 1,200 / 2,000 \mathrm{mg}$, or $1,600 / 800$ mg. ${ }^{34}$ Three patients were enrolled in each cohort and received treatment every 3 weeks for a total of nine infusions. Patients were premedicated with diphenhydramine and acetaminophen; corticosteroids were permitted at investigator discretion for patients with a large tumor burden, previous severe reactions to rituximab, or severe infusion-related reactions (IRRs) observed with the first obinutuzumab infusion. IRRs were predominantly associated with the first infusion, and resolved with slowing or interruption of the infusion and/or steroid administration, with patients ultimately able to receive their full dose. Elevated cytokine levels were observed after the first infusion, becoming less common with subsequent infusions. No dose-limiting toxicities were identified. All responses were noted in the FL cohort $(n=13)$, with an ORR of $69 \%(n=9)$ and five patients $(38 \%)$ achieving a complete response $(\mathrm{CR})$. Two responses were noted in rituximabrefractory $\mathrm{FL}$, with rituximab-refractory defined as no response or response duration $<6$ months to prior rituximabcontaining therapy. A similar safety and tolerability profile was described in a multicenter, dose escalation Phase I trial of 12 Japanese patients with relapsed/refractory indolent CD20+ B-cell NHL. ${ }^{35}$ IRR was the most frequently observed adverse event (AE) and the most commonly associated with first infusion, and no dose-limiting toxicities were identified. ${ }^{35}$ Of the eight patients with FL, six (75\%) responded, of whom two achieved a CR. Furthermore, one of the two rituximab-refractory patients achieved a response, and both experienced reduction in tumor mass.

The Phase I GAUSS trial evaluated 22 patients with relapsed/refractory CD20 ${ }^{+} \mathrm{NHL}$ or CLL who received obinutuzumab monotherapy at doses of $100 / 200 \mathrm{mg}, 200 / 400$ $\mathrm{mg}, 400 / 800 \mathrm{mg}, 800 / 1,200 \mathrm{mg}, 1,200 / 2,000 \mathrm{mg}$, and $1,000 / 1,000 \mathrm{mg}$ once weekly for up to 4 weeks for induction therapy, followed by obinutuzumab maintenance treatment of one dose every 3 months, for a maximum of eight infusions. ${ }^{36}$ Of the 19 (86\%) patients who received at least one prior rituximab-containing regimen (median two, range: one to four), 13 were refractory to rituximab (no response or progression within 6 months of treatment). Low-grade infections were observed in $32 \%$ of patients during induction and in five of eight patients during the maintenance phase. The only grade 3/4 infection reported occurred during maintenance, characterized as a grade 3 respiratory infection and sinusitis. Of the eight patients who went on to receive maintenance treatment, there were no discontinuations for treatment-related toxicity. In the subset of patients with FL, the ORR was $40 \%(n=10$, including one CR and three partial responses [PRs]). The duration of response ranged from 3 to 21.1 months, and four of seven responses lasted more than a year.

\section{Phase II}

In the Phase II GAUGUIN study, two dosing regimens of obinutuzumab were evaluated in relapsed/refractory DLBCL, mantle-cell lymphoma, and indolent NHL. ${ }^{37}$ The lower dose regimen consisted of $400 \mathrm{mg}$ on $\mathrm{C} 1 \mathrm{D} 1$ and D8 and C2-C8D1, whereas the higher dose regimen consisted of 1,600 $\mathrm{mg}$ on C1D1 and D8 and C2-8D1 $800 \mathrm{mg}$. Fourteen FL patients received the lower dose regimen and 20 received the higher dose. The majority had previously received rituximab (17 of 18 in the $400 / 400 \mathrm{mg}$ arm; 21 of 22 in the 1,600/800 mg arm), with a median of two previous lines of rituximab-based therapy. Twelve of 18 patients in the $400 / 400 \mathrm{mg}$ arm and ten of 22 in the 1,600/800 mg arm were defined as rituximabrefractory. Among the rituximab-refractory patients, seven in the lower dose arm and seven in the higher dose arm were unresponsive to prior rituximab therapy, and five and three had relapsed within 6 months after rituximab therapy, respectively. In the higher dose group $(1,600 / 800 \mathrm{mg})$, an ORR of 55\% was achieved (CR 9\%), with a median PFS of 11.9 months. The ORR in rituximab-refractory patients was $50 \%$ in the higher dose treatment arm compared to $17 \%$ in the lower dose arm. The results were encouraging, particularly for rituximab-refractory patients. 
Obinutuzumab was compared directly to rituximab in the open-label, multicenter, randomized Phase II GAUSS trial in which 175 patients with relapsed $\mathrm{CD} 20^{+}$indolent NHL received obinutuzumab or rituximab monotherapy for induction followed by 2 years of maintenance therapy in nonprogressing patients. ${ }^{38}$ Of the 175 patients, 149 had FL (obinutuzumab, $n=74$; rituximab, $n=75$ ). Patients had received a median of two prior lines of therapy (obinutuzumab, range: one to seven; rituximab, range: one to six). Induction consisted of four once-weekly intravenous infusions of obinutuzumab $(1,000 \mathrm{mg})$ or rituximab $\left(375 \mathrm{mg} / \mathrm{m}^{2}\right)$. After induction, patients with a CR, PR, or stable disease were eligible to receive maintenance therapy every 2 months for up to 2 years or until progression with the same $\mathrm{mAb}$ and dose as administered during induction. By investigator assessment, the ORR for the FL patients trended higher in the obinutuzumab arm (44.6\%) compared to the rituximab arm $(33.3 \%)(P=0.08)$. Nine patients in the obinutuzumab $\operatorname{arm}(12.2 \%)$ and four in the rituximab arm (5.3\%) achieved $\mathrm{CR}$ or $\mathrm{CR}$ unconfirmed; this difference was not statistically significant $(P=0.07)$. Independent review committee also found the ORR to be higher with obinutuzumab versus rituximab ( $44.6 \%$ vs $26.7 \%, P=0.01$ ), but with no difference in $\mathrm{CR} / \mathrm{CR}$-unconfirmed rate (5.4 vs $4, P=0.34$ ). However, the higher ORR seen with obinutuzumab did not ultimately translate into improvement in PFS at a median follow-up of 32 months. The similar PFS rates in this Phase II study may be attributable to study sample size or prior treatment regimens. Alternatively, it may highlight the fact that the true benefit of obinutuzumab lies in combination with chemotherapy, as opposed to single-agent activity.

\section{Obinutuzumab combination trials}

\section{Combination with chemotherapy}

Phase IB

Like rituximab, obinutuzumab has been explored in combination with chemotherapy. The Phase IB GAUDI study evaluated the safety and activity of obinutuzumab combined with either cyclophosphamide, doxorubicin, vincristine, and prednisone (O-CHOP; every 3 weeks for six to eight cycles) or fludarabine and cyclophosphamide (O-FC; every 4 weeks for four to six cycles) in 56 patients with relapsed/ refractory FL. ${ }^{39}$ Obinutuzumab was given according to either a 1,600/800 mg schedule (1,600 mg on D1 and D8 of C1;800 mg on D1 of subsequent cycles) or a 400/400 $\mathrm{mg}$ schedule in each arm, in addition to standard doses of chemotherapy. Enrolled patients who responded to treatment were eligible for maintenance therapy every 3 months up to 2 years. Patients had received a median of one prior line of rituximab therapy and $14 \%$ of O-CHOP patients and $36 \%$ of O-FC patients had rituximab-refractory disease. The end-of-induction ORR for O-CHOP was $93 \%$ in the $400 / 400 \mathrm{mg}$ arm $(14 \% \mathrm{CR})$ and $100 \%$ in the $1,600 / 800 \mathrm{mg}$ arm $(64 \% \mathrm{CR})$. In comparison, the end-of-induction ORR for O-FC was $100 \%$ in the $400 / 400 \mathrm{mg}$ arm (79\% CR) and $86 \%$ in the $1,600 / 800 \mathrm{mg}$ arm $(21 \% \mathrm{CR})$. Furthermore, all 14 patients with rituximab-refractory disease experienced a response, with four CRs (one from O-CHOP and three from O-FC). This study was the first to demonstrate the benefit of obinutuzumab-based chemoimmunotherapy in rituximabrefractory disease.

Obinutuzumab was also evaluated in combination with CHOP or bendamustine in patients with previously untreated FL, with a similar safety profile in which neither an obinutuzumab-associated dose-limiting toxicity nor unexpected AE was seen. ${ }^{40}$ In the most recent subset analysis of the Phase IB GAUDI study, 81 previously untreated FL patients received either obinutuzumab (1,000 mg intravenously on D1 and D8 of $\mathrm{C} 1$ and D1 of subsequent cycles) plus bendamustine (O-B) or $\mathrm{CHOP}^{41}$ All patients experienced at least one $\mathrm{AE}$ during induction; 64\% (O-B 5\%, O-CHOP 78\%) experienced grade $3 / 4$ adverse reactions. The most common grade $3 / 4$ hematologic $\mathrm{AE}$ was neutropenia, occurring in $36 \%$ of patients (O-B 29\%, O-CHOP 43\%) during induction. The most common nonhematologic $\mathrm{AE}$ was infection, with eleven patients (O-B six, O-CHOP five) experiencing grade 3 infection and one patient in the O-B group experiencing grade 4 neutropenic infection. Dose delays were required in $65 \%$ of patients (O-B 59\%, O-CHOP 73\%). B-cell populations decreased rapidly after the first obinutuzumab infusion, with all patients showing B-cell depletion throughout treatment. The median time from end of treatment to B-cell recovery was 24 months for the O-B group and 29 months for the O-CHOP group. Median levels of T cells and NK cells were also low throughout induction committee, with full recovery to baseline during maintenance. In terms of efficacy, at the end of induction, ORRs and CR rates were similar (O-B 93\% and $37 \%$, respectively; O-CHOP $95 \%$ and $35 \%$, respectively). The estimated PFS at 36 months was $90 \%$ in the O-B arm and $84 \%$ in the O-CHOP arm.

\section{Phase III}

Based on the clinical potential of obinutuzumab in combination with chemotherapy, a number of Phase III studies have been conducted in various disease settings. The GADOLIN trial was the first randomized Phase III study to show that an 
alternative anti-CD20 mAb is clinically useful for patients with rituximab-refractory indolent NHL. In this open label, randomized, multicenter study, the safety and efficacy of O-B followed by obinutuzumab maintenance was evaluated in comparison to treatment with bendamustine induction monotherapy (B arm) in rituximab-refractory indolent NHL. ${ }^{42}$ Patients were considered eligible if they had not experienced a response to a rituximab-containing regimen or if they had experienced a progression during or within 6 months of a receiving rituximab as a part of induction or maintenance. The majority of patients (321 of 394) had FL. Patients had received a median of two prior treatment regimens. Patients considered refractory to rituximab chemoimmunotherapy and rituximab monotherapy were evenly distributed between the arms. The percentage of patients who did not respond to or progressed during/within 6 months of the last dose of chemoimmunotherapy induction was similar between the arms as well (49\% O-B, 55\% B). Patients in the O-B arm were administered obinutuzumab 1,000 mg intravenously on D1, D8, and D15 of C1 and D1 of C2-C6, plus bendamustine 90 $\mathrm{mg} / \mathrm{m}^{2}$ per day intravenously on D1 and D2 of each 28-day cycle for up to six cycles, whereas patients in the B arm received single-agent bendamustine at $120 \mathrm{mg} / \mathrm{m}^{2}$. Patients in the O-B group without evidence of progression following induction received obinutuzumab maintenance 1,000 mg every 2 months for 2 years or until disease progression. Tumor response was assessed before cycle 4 of induction, 28-42 days after last induction dose, then every 3 months for 2 years, and then every 6 months for an additional 2 years or until progression. In the primary analysis, the median independent review committee-assessed PFS was longer in the O-B arm (194 patients, median not reached) than in the $\mathrm{B}$ arm (202 patients, 14.9 months) with a $45 \%$ reduction in risk of progression or death. PFS favored the addition of the obinutuzumab, regardless of whether disease was considered refractory to rituximab monotherapy, rituximab-based chemoimmunotherapy induction, or maintenance rituximab after chemotherapy-containing induction. Based on the results of this trial, FDA approval was most recently granted for obinutuzumab in the treatment of FL that has relapsed after or was refractory to a rituximab-containing regimen.

The GADOLIN data were updated with 10 months of additional follow-up and 17 more patients. ${ }^{43}$ The safety profile was consistent with that of the primary analysis. There were more grade $>3$ AEs with O-B (72.5\%) compared to the B arm $(65.5 \%)$, with mainly neutropenia ( $34.8 \%$ vs $27.1 \%)$, anemia (7.4\% vs $10.8 \%$ ), thrombocytopenia ( $10.8 \%$ vs $15.8 \%)$, and IRRs (9.3\% vs 3.5\%). There was a similar incidence of grade $>3$ infections $(22.5 \%$ in O-B arm vs $19.2 \%$ in B arm) and secondary malignancies (5.9\% vs 5.4\%). After a median follow-up of 31 months, the investigator-assessed median PFS for the FL cohort was 25 months in the O-B arm compared to 14 months in the B arm. The median OS had not been reached in the O-B arm at the time of analysis compared to 54 months $(P=0.0061)$; fewer patients died in the O-B arm (24\%) than in the B arm (37\%). As such, the updated analysis confirms the previously reported improvement in PFS and demonstrated the OS benefit of obinutuzumab-based chemotherapy in rituximab-refractory disease. Whether the subset of enrolled patients who had experienced a relapse during or within 6 months of a rituximab-containing regimen may have benefited from additional rituximab, as opposed to obinutuzumab, was not addressed by this study. However, the potential for delayed neutropenia, hypogammaglobulinemia, and subsequent infections favors the use of the newer antibody over further administration of rituximab.

Given the promising results of the early-phase trials, the Phase III GALLIUM study compared obinutuzumab chemotherapy followed by obinutuzumab maintenance (O-chemo) to rituximab chemotherapy with rituximab maintenance (R-chemo) for first-line treatment in NHL. ${ }^{44}$ Results were assessed for 1,202 FL patients. Treatment arms were randomized and balanced by disease stage and FL International Prognostic Index (FLIPI) score. The end-of-induction ORRs by computed tomography were similar between the arms (O-chemo 88.5\%, R-chemo 86.9\%). However, positron-emission tomography (PET) scan assessments were not available at the time of presentation. Of note, $92 \%$ of patients achieved minimal residual disease negativity in the $\mathrm{O}$-chemo arm compared to $85 \%$ in the $\mathrm{R}$-chemo arm $(P=0.0041)$, suggesting that the true $C R$ rates may have differed between the arms. The primary end point of the study was PFS by investigator assessment for the FL population: the 3-year PFS was $80 \%$ with O-chemo vs $73 \%$. The difference remained statistically significant when analyzed by independent review $(P=0.0138)$. At a median follow-up of 34.5 (range: 0-54.5) months, there was a $34 \%$ reduction in the risk of progression or death. Similarly, the time to next treatment favored the obinutuzumab $\operatorname{arm}(87 \%$ vs $81 \%, P=0.0094)$. However, there was no difference in OS at 3 years $(P=0.21)$. O-chemo patients had a slightly higher frequency of grade $3-5$ AEs $(74.6 \%$ compared to $67.8 \%$ ); the incidence of treatment discontinuation in the absence of disease progression was relatively similar between the arms. Obinutuzumab-based immunochemotherapy and maintenance resulted in clinically meaningful improvement in PFS compared to rituximab-based therapy. These data 
support O-chemo becoming a new standard of care in previously untreated patients with FL.

\section{Combination with targeted therapies Lenalidomide}

There has been a concerted effort to investigate the utility of targeted therapies, which in theory possess less nonspecific toxicity compared to conventional chemotherapeutics, for the treatment of FL. Like rituximab, obinutuzumab has also been explored in combination with a number of novel agents. Based on the success of the rituximab with the immunomodulatory agent lenalidomide in previously untreated FL $(\text { ORR }>90 \%)^{45}$ and the upcoming phase III RELEVANCE study of the doublet in comparison to R-chemotherapy (NCT01650701), ${ }^{46}$ Morschhauser et al conducted the Phase I GALEN trial of obinutuzumab plus lenalidomide in patients with relapsed or refractory $\mathrm{CD} 20^{+} \mathrm{FL}(\mathrm{n}=20) .{ }^{47}$ Patients had received a median of two prior systemic therapies, and eight were rituximab-refractory. The most common AEs (>20\%) were neutropenia (53\%), constipation (53\%), upper respiratory infection (37\%, seven of 19$)$ and asthenia (37\%, seven of 19). Four dose-limiting toxicities occurred in two patients, which were deemed unrelated to study treatment: one unexplained death in the setting of worsening grade 3 pleural effusion, and one grade 3 pulmonary infection in the setting of grade 3 hypokalemia. Thirteen (68\%) patients achieved a response, including seven CRs. The recommended dose for the combination of lenalidomide with obinutuzumab was established at $20 \mathrm{mg}$, and is currently being investigated in the ongoing Phase II portion of the study in relapsed/ refractory FL (NCT01582776). ${ }^{48}$

Fowler et al also studied the antibody in combination with escalating doses of lenalidomide (10 mg, $15 \mathrm{mg}$, and $20 \mathrm{mg}$ ) and found similar results in 15 patients with relapsed small LL, marginal lymphoma, and FL grades 1-3A (21\% had low, 29\% intermediate, and 50\% high FLIPI scores). ${ }^{49}$ No dose-limiting toxicities were observed in the frontline setting. The most common nonhematologic toxicities were fatigue $(80 \%, 12$ of 15$)$, constipation $(60 \%, 9$ of 15$)$ and diarrhea (47\%, 7 of 15 ), and $20 \%$ of patients experienced neutropenia. An encouraging ORR of $93 \%$ was noted, although only four patients achieved a CR (27\%). The doublet followed by maintenance obinutuzumab is also being investigated in previously untreated disease (NCT02871219). ${ }^{50}$

\section{B-cell-receptor antagonists}

As in CLL, B-cell-receptor antagonists such as idelalisib and ibrutinib, have been explored extensively in combination with anti-CD20 antibodies in FL. After the approval of idelalisib, the second-generation PI3K inhibitor duvelisib was combined with obinutuzumab (DO) or rituximab (DR) in the frontline Phase I/IIB CONTEMPO study.$^{51}$ In general, the combination of duvelisib $25 \mathrm{mg}$ twice daily with rituximab or obinutuzumab was well tolerated in these patients. Both the DR and DO arms were continued to Phase II of the study, which is ongoing. Preliminary clinical activity was noted in both arms (DR, ORR 87\%, CR 22\%; DO, ORR 91\%, CR 18\%). Similarly, the Bruton tyrosine-kinase inhibitor ibrutinib is being combined with obinutuzumab in previously untreated FL patients (NCT02689869). ${ }^{52}$ These studies support the role of dual B-cell-directed therapy in FL.

\section{Immune-checkpoint blockade}

The immunological interplay between malignant lymphocytes and the immune cells of the tumor microenvironment has become an attractive target in FL. Antitumor responses may be induced by usurping immune-checkpoint pathways, such as PD1 pathways. ${ }^{53-55}$ mAbs directed against the PD receptor or its ligands are felt to reverse the tumor-induced downregulation of T-cell function and augment antitumor immune activity. This has led to rituximab being explored in combination with the anti-PD1 antibody pidilizumab, yielding a $66 \%$ ORR and $52 \% \mathrm{CR}$ rate in relapsed patients. ${ }^{56}$ Obinutuzumab has recently been explored with the anti PDL1 antibody atezolizumab in FL. In a multicenter open-label Phase IB trial, atezolizumab was combined with obinutuzumab in six patients with relapsed or refractory FL $(n=3)$ and DLBCL $(n=3)$ (NCT02220842). ${ }^{57}$ The median number of prior therapies was four (range: 2-6), and all patients had received at last one prior rituximab-containing therapy regimen. Patients received the doublet for eight 3-week cycles followed by an additional 6 months of atezolizumab. There were three grade 3 AEs of thrombocytopenia and ileus in three patients. Preliminary efficacy results showed that after four cycles of therapy, two achieved a PR, including one FL patient. Expansion cohorts are currently enrolling.

\section{Venetoclax}

The selective BCL2 inhibitor venetoclax, currently approved in previously treated CLL with deletion $17 \mathrm{p}$, is under investigation in FL as well. As a single agent in a relapsed/refractory Phase I study, venetoclax produced an ORR of $38 \%$ and median PFS of 11 months. ${ }^{58}$ It was subsequently combined with R-CHOP and O-CHOP in a Phase IB study in 56 patients with B-cell NHL, primarily as a frontline regimen. ${ }^{59}$ Of those enrolled, $43 \%$ had FL, 30\% had DLBCL, and 9\% had marginal-zone lymphoma. The most common AEs in both arms were neutropenia (46\%), nausea (45\%), fatigue (38\%), 
and diarrhea (36\%). Of the 42 patients in the intent-to-treat analysis that were evaluable for efficacy, 18 of $21(85.7 \%)$ in venetoclax plus O-CHOP and 17 of 21 (81\%) in venetoclax plus R-CHOP had a response. Ongoing Phase I studies include obinutuzumab with venetoclax in previously untreated FL and venetoclax and lenalidomide in relapsed and refractory B-cell NHL (NCT02877550 and NCT02992522). ${ }^{60,61}$

\section{Combination with antibody-drug conjugates}

Since the success of brentuximab, a number of B-celldirected antibody-drug conjugates have been in development. Of interest is polatuzumab vedotin, which consists of an anti-CD79b mAb linked to the antitubulin molecule monomethyl auristatin E. Early results from an ongoing Phase IB/II study of polatuzumab vedotin at $1.8 \mathrm{mg} / \mathrm{kg}$ in combination with obinutuzumab in patients with relapsed/refractory DLBCL and FL indicated both tolerability and efficacy. ${ }^{62}$ When bendamustine was added to the doublet in relapsed and refractory FL, the ORR was $100 \% ; 67 \%$ (8 of 12 ) had grade 3/4 AEs, with the most common being neutropenia (33\%) and thrombocytopenia (17\%). ${ }^{63}$ A combination with obinutuzumab and venetoclax in relapsed and refractory FL is under way (NCT02611323). ${ }^{64}$

\section{Safety}

The clinical safety of obinutuzumab has been pooled from a number of clinical studies. No dose-limiting toxicity has been observed, despite administration of doses up to 2,000 mg. The major safety events observed have been neutropenia, infections, and IRRs, none of which is related to obinutuzumab dose or exposure. ${ }^{65}$ The most common adverse reactions of all grades ( $>10 \%$ ) were IRRs, neutropenia, thrombocytopenia, anemia, pyrexia, cough, and musculoskeletal disorder. ${ }^{66}$ The most common grade $3-4$ adverse reactions ( $>10 \%$ ) were IRRs, neutropenia, and thrombocytopenia. Infusion reactions occurred in $69 \%$ of patients receiving obinutuzumab; $21 \%$ experienced grade $3 / 4$ reactions. The incidence of IRRs appears to be higher with obinutuzumab (all grades $74 \%$, grade $3 / 411 \%$ ) than rituximab (all grades $51 \%$, grade $3 / 4$ $5 \%) .{ }^{38}$ As such, premedication with high doses of glucocorticoid, in addition to acetaminophen and antihistamine, is recommended prior to initial obinutuzumab infusions and subsequently as needed.

In the GALLIUM study, the obinutuzumab arm had more frequently reported grade 3 AEs $(74.6 \%)$ compared to the rituximab arm (67.8\%), with $16.3 \%$ of patients in the obinutuzumab arm discontinuing treatment compared to $14.2 \%$ of patients in the rituximab arm discontinuing treatment. ${ }^{44}$ The most common grade 3 or higher AEs were neutropenia
(43.9\% O-chemo vs 37.9\% R-chemo), febrile neutropenia (6.9\% O-chemo vs 4.9\% R-chemo), and thrombocytopenia (6.1\% O-chemo vs $2.7 \%$ R-chemo). The potential mechanism of IRRs has been thought to be related to increased cytokine release, including IL-8, IL-6, and TNF $\alpha .{ }^{67-71}$ Cytokine levels were significantly elevated after first obinutuzumab infusion, suggesting that IRRs may be related to cytokine release from malignant $\mathrm{B}$ cells rather than CDC. Multivariate analysis by Freeman et al supports the idea that higher rates of IRR seen with obinutuzumab result from enhanced binding of anti-CD20 mAbs to both malignant B cells and FcyRIIIaexpressing effector cells, leading to greater cytotoxicity. ${ }^{67}$ Early- and late-onset neutropenia has been reported with greater incidence in obinutuzumab compared to rituximab; SDF1/CLCX12 is implicated in late-onset neutropenia. ${ }^{72}$ Fatal infections have also been reported, including serious bacterial, fungal, and new or reactivated viral infections.

Hepatitis B reactivation, in some cases leading to fulminant hepatitis, can happen in patients receiving a CD20 mAb, including obinutuzumab. All patients must be screened with $\mathrm{HBsAg}$ and anti-HBc before initiating treatment with obinutuzumab. Progressive multifocal leukoencephalopathy can also occur in patients receiving obinutuzumab. Tumor lysis syndrome has also been reported in patients receiving obinutuzumab; therefore, antihyperuricemics should be considered for patients with high tumor burden, high circulating absolute lymphocyte counts $>25 \times 10^{9} / \mathrm{L}$, or renal impairment, in addition to adequate hydration. Obinutuzumab has not been studied in patients with baseline creatinine clearance $<30 \mathrm{~mL} / \mathrm{min}$, nor has it been studied in patients with hepatic impairment. ${ }^{66}$

\section{Conclusion}

Obinutuzumab is the first humanized glycoengineered $\operatorname{IgG}_{1}$ anti-CD20 mAb to be tested in clinical trials for NHL. Its mechanism of intratetramer binding to mediate apoptotic induction and antibody-dependent cellular cytotoxicity has proven to have greater efficacy than rituximab in head-tohead studies and in rituximab-refractory FL. While some may question the appropriateness of such a high dose of bendamustine $\left(120 \mathrm{mg} / \mathrm{m}^{2}\right)$ in the comparator arm of the GADOLIN study, obinutuzumab was granted approval by the FDA for combination with bendamustine $(90 \mathrm{mg} /$ $\mathrm{m}^{2}$ ) in patients who have relapsed after or are refractory to rituximab, based on the improvement in PFS and more recently OS.

As the bendamustine-rituximab regimen has progressively become the frontline standard of care for FL based on the results of the STIL NHL1 and BRIGHT studies, identifying the appropriate patient for the obinutuzumab-bendamustine com- 
bination can be difficult. Retreatment with bendamustine can be associated with significant and often persistent myelosuppression. Whether this remains an ongoing issue is dependent on two ongoing frontline Phase III trials: the GALLIUM and RELEVANCE studies. As previously mentioned, the GALLIUM study demonstrated increased PFS and rates of minimal residual disease negativity with $\mathrm{O}$-chemo in comparison to R-chemo. These findings indicate that there may be a difference in positron-emission tomography-derived CRs, which has been associated with an improvement in OS, although not yet evident at 3 years of follow-up. ${ }^{11,73}$ Nevertheless, these data would support a frontline approval of obinutuzumab in combination with chemotherapy. However, given the well-established lengthy PFS with R-chemo and increased costs associated with the newer antibody, rituximab-based chemoimmunotherapy may remain a standard in much of the world.

The RELEVANCE study challenges whether chemotherapy should even be used in the frontline treatment of FL, based on encouraging Phase II data of the rituximablenalidomide combination. If the doublet gains an upfront indication, obinutuzumab may replace rituximab, based on previously demonstrated superiority in combination with chemotherapy and tolerability with lenalidomide. A greater understanding of the multiple factors contributing to the pathogenesis of FL and the increasing availability of novel agents enables the reevaluation of current therapeutic directives. These developments are highly encouraging, and justify the expectation that the management of patients with FL will further improve within the coming years.

\section{Disclosure}

The authors report no conflicts of interest in this work.

\section{References}

1. Howlader N, Morton LM, Feuer EJ, Besson C, Engels EA. Contributions of subtypes of non-Hodgkin lymphoma to mortality trends. Cancer Epidemiol Biomarkers Prev. 2016;25(1):174-179.

2. Jaffe ES. The 2008 WHO classification of lymphomas: implications for clinical practice and translational research. Hematology Am Soc Hematol Educ Program. 2009:523-531.

3. van Meerten T, Hagenbeek A. CD20-targeted therapy: a breakthrough in the treatment of non-Hodgkin's lymphoma. Neth J Med. 2009;67(7): 251-259.

4. Cragg MS, Morgan SM, Chan HT, et al. Complement-mediated lysis by anti-CD20 mAb correlates with segregation into lipid rafts. Blood. 2003;101(3):1045-1052.

5. Reff ME, Carner K, Chambers KS, et al. Depletion of B cells in vivo by a chimeric mouse human monoclonal antibody to CD20. Blood. 1994;83(2):435-445.

6. Herold M, Haas A, Srock S, et al. Rituximab added to first-line mitoxantrone, chlorambucil, and prednisolone chemotherapy followed by interferon maintenance prolongs survival in patients with advanced follicular lymphoma: an East German Study Group Hematology and Oncology Study. J Clin Oncol. 2007;25(15):1986-1992.
7. Hiddemann W, Kneba M, Dreyling M, et al. Frontline therapy with rituximab added to the combination of cyclophosphamide, doxorubicin, vincristine, and prednisone (CHOP) significantly improves the outcome for patients with advanced-stage follicular lymphoma compared with therapy with $\mathrm{CHOP}$ alone: results of a prospective randomized study of the German Low-Grade Lymphoma Study Group. Blood. 2005;106(12):3725-3732.

8. Marcus R, Imrie K, Belch A, et al. CVP chemotherapy plus rituximab compared with CVP as first-line treatment for advanced follicular lymphoma. Blood. 2005;105(4):1417-1423.

9. Marcus R, Imrie K, Solal-Celigny P, et al. Phase III study of R-CVP compared with cyclophosphamide, vincristine, and prednisone alone in patients with previously untreated advanced follicular lymphoma. J Clin Oncol. 2008;26(28):4579-4586.

10. Salles G, Mounier N, de Guibert S, et al. Rituximab combined with chemotherapy and interferon in follicular lymphoma patients: results of the GELA-GOELAMS FL2000 study. Blood. 2008;112(13):4824-4831.

11. Rummel MJ, Niederle N, Maschmeyer G, et al. Bendamustine plus rituximab versus $\mathrm{CHOP}$ plus rituximab as first-line treatment for patients with indolent and mantle-cell lymphomas: an open-label, multicentre, randomised, phase 3 non-inferiority trial. Lancet. 2013;381(9873):1203-1210.

12. Flinn IW, van der Jagt R, Kahl BS, et al. Randomized trial of bendamustine-rituximab or R-CHOP/R-CVP in first-line treatment of indolent NHL or MCL: the BRIGHT study. Blood. 2014;123(19):2944-2952.

13. Federico M, Luminari S, Dondi A, et al. R-CVP versus R-CHOP versus R-FM for the initial treatment of patients with advanced-stage follicular lymphoma: results of the FOLL05 trial conducted by the Fondazione Italiana Linfomi. J Clin Oncol. 2013;31(12):1506-1513.

14. Cartron G, Dacheux L, Salles G, et al. Therapeutic activity of humanized anti-CD20 monoclonal antibody and polymorphism in IgG Fc receptor FcyRIIIa gene. Blood. 2002;99(3):754-758.

15. van Meerten T, van Rijn RS, Hol S, Hagenbeek A, Ebeling SB. Complement-induced cell death by rituximab depends on CD20 expression level and acts complementary to antibody-dependent cellular cytotoxicity. Clin Cancer Res. 2006;12(13):4027-4035.

16. Czuczman MS, Olejniczak S, Gowda A, et al. Acquirement of rituximab resistance in lymphoma cell lines is associated with both global CD20 gene and protein down-regulation regulated at the pretranscriptional and posttranscriptional levels. Clin Cancer Res. 2008;14(5):1561-1570.

17. Wierda WG, Kipps TJ, Mayer J, et al. Ofatumumab as single-agent CD20 immunotherapy in fludarabine-refractory chronic lymphocytic leukemia. J Clin Oncol. 2010;28(10):1749-1755.

18. Teeling JL, Mackus WJ, Wiegman LJ, et al. The biological activity of human CD20 monoclonal antibodies is linked to unique epitopes on CD20. J Immunol. 2006;177(1):362-371.

19. Hagenbeek A, Gadeberg O, Johnson P, et al. First clinical use of ofatumumab, a novel fully human anti-CD20 monoclonal antibody in relapsed or refractory follicular lymphoma: results of a phase $1 / 2$ trial. Blood. 2008;111(12):5486-5495.

20. Czuczman MS, Fayad L, Delwail V, et al. Ofatumumab monotherapy in rituximab-refractory follicular lymphoma: results from a multicenter study. Blood. 2012;119(16):3698-3704.

21. Niederfellner G, Lammens A, Mundigl O, et al. Epitope characterization and crystal structure of GA101 provide insights into the molecular basis for type I/II distinction of CD20 antibodies. Blood. 2011; 118(2):358-367.

22. Klein C, Lammens A, Schäfer W, et al. Epitope interactions of monoclonal antibodies targeting CD20 and their relationship to functional properties. MAbs. 2013;5(1):22-33.

23. Alduaij W, Ivanov A, Honeychurch J, et al. Novel type II anti-CD20 monoclonal antibody (GA101) evokes homotypic adhesion and actindependent, lysosome-mediated cell death in B-cell malignancies. Blood. 2011;117(17):4519-4529.

24. Honeychurch J, Alduaij W, Azizyan M, et al. Antibody-induced nonapoptotic cell death in human lymphoma and leukemia cells is mediated through a novel reactive oxygen species-dependent pathway. Blood. 2012;119(15):3523-3533. 
25. Ashraf SQ, Umana P, Mössner E, et al. Humanised IgG1 antibody variants targeting membrane-bound carcinoembryonic antigen by antibody-dependent cellular cytotoxicity and phagocytosis. Br J Cancer. 2009;101(10):1758-1768.

26. Mössner E, Brünker P, Moser S, et al. Increasing the efficacy of CD20 antibody therapy through the engineering of a new type II anti-CD20 antibody with enhanced direct and immune effector cell-mediated B-cell cytotoxicity. Blood. 2010;115(22):4393-4402.

27. Liu SD, Chalouni C, Young JC, Junttila TT, Sliwkowski MX, Lowe JB. Afucosylated antibodies increase activation of Fc $\gamma$ RIIIa-dependent signaling components to intensify processes promoting ADCC. Cancer Immunol Res. 2015;3(2):173-183.

28. Herter S, Herting F, Mundigl O, et al. Preclinical activity of the type II CD20 antibody GA101 (obinutuzumab) compared with rituximab and ofatumumab in vitro and in xenograft models. Mol Cancer Ther. 2013;12(10):2031-2042.

29. Dalle S, Reslan L, de Horts TB, et al. Preclinical studies on the mechanism of action and the anti-lymphoma activity of the novel anti-CD20 antibody GA101. Mol Cancer Ther. 2011;10(1):178-185.

30. Decaup E, Jean C, Laurent C, et al. Anti-tumor activity of obinutuzumab and rituximab in a follicular lymphoma 3D model. Blood Cancer J. 2013;3:e131.

31. Herting F, Friess T, Bader S, et al. Enhanced anti-tumor activity of the glycoengineered type II CD20 antibody obinutuzumab (GA101) in combination with chemotherapy in xenograft models of human lymphoma. Leuk Lymphoma . 2014;55(9):2151-2160.

32. Kern DJ, James BR, Blackwell S, Gassner C, Klein C, Weiner GJ. GA101 induces NK-cell activation and antibody-dependent cellular cytotoxicity more effectively than rituximab when complement is present. Leuk Lymphoma. 2013;54(11):2500-2505.

33. Cartron G, Hourcade-Potelleret F, Morschhauser F, et al. Rationale for optimal obinutuzumab/GA101 dosing regimen in B-cell non-Hodgkin lymphoma. Haematologica. 2016;101(2):226-234.

34. Salles G, Morschhauser F, Lamy T, et al. Phase 1 study results of the type II glycoengineered humanized anti-CD20 monoclonal antibody obinutuzumab (GA101) in B-cell lymphoma patients. Blood. 2012;119(22):5126-5132.

35. Ogura M, Tobinai K, Hatake K, et al. Phase I study of obinutuzumab (GA101) in Japanese patients with relapsed or refractory B-cell nonHodgkin lymphoma. Cancer Sci. 2013;104(1):105-110.

36. Sehn LH, Assouline SE, Stewart DA, et al. A phase 1 study of obinutuzumab induction followed by 2 years of maintenance in patients with relapsed CD20-positive B-cell malignancies. Blood. 2012;119(22):5118-5125

37. Salles GA, Morschhauser F, Solal-Celigny P, et al. Obinutuzumab (GA101) in patients with relapsed/refractory indolent non-Hodgkin lymphoma: results from the phase II GAUGUIN study. J Clin Oncol. 2013;31(23):2920-2926.

38. Sehn LH, Goy A, Offner FC, et al. Randomized phase II trial comparing obinutuzumab (GA101) with rituximab in patients with relapsed CD20+ Indolent B-cell non-Hodgkin lymphoma: final analysis of the GAUSS study. J Clin Oncol. 2015;33(30):3467-3474.

39. Radford J, Davies A, Cartron G, et al. Obinutuzumab (GA101) plus CHOP or FC in relapsed/refractory follicular lymphoma: results of the GAUDI study (BO21000). Blood. 2013;122(7):1137-1143.

40. Dyer MJ, Grigg AP, Díaz MG, et al. Obinutuzumab (GA101) in combination with CHOP (cyclophosphamide, doxorubicin, vincristine and prednisone) or bendamustine for the first-line treatment of follicular non-Hodgkin lymphoma: final results from the maintenance phase of the phase IB GAUDI study. Blood. 2014;124(21):1743.

41. Grigg A, Dyer MJ, Díaz MG, et al. Safety and efficacy of obinutuzumab with CHOP or bendamustine in previously untreated follicular lymphoma. Haematologica. 2016;2016:152272.

42. Sehn LH, Chua N, Mayer J, et al. Obinutuzumab plus bendamustine versus bendamustine monotherapy in patients with rituximab-refractory indolent non-Hodgkin lymphoma (GADOLIN): a randomised, controlled, open-label, multicentre, phase 3 trial. Lancet Oncol. 2016; 17(8):1081-1093
43. Cheson BD, Trněný $\mathrm{M}$, Bouabdallah $\mathrm{K}$, et al. Obinutuzumab plus bendamustine followed by obinutuzumab maintenance prolongs overall survival compared with bendamustine alone in patients with rituximabrefractory indolent non-Hodgkin lymphoma: updated results of the GADOLIN study. Blood. 2016;128(22):615.

44. Marcus RE, Davies AJ, Ando K, et al. Obinutuzumab-based induction and maintenance prolongs progression-free survival (PFS) in patients with previously untreated follicular lymphoma: primary results of the randomized phase 3 GALLIUM study. Blood. 2016;128(22):6.

45. Fowler NH, Davis RE, Rawal S, et al. Safety and activity of lenalidomide and rituximab in untreated indolent lymphoma: an open-label, phase 2 trial. Lancet Oncol. 2014;15(12):1311-1318.

46. Lymphoma Academic Research Organisation. A phase 3 open label randomized study to compare the efficacy and safety of rituximab plus lenalidomide (CC-5013) versus rituximab plus chemotherapy followed by rituximab in subjects with previously untreated follicular lymphoma (RELEVANCE). Available from: https:/clinicaltrials.gov/ct2/show/ NCT01650701. NLM identifier: NCT01650701. Accessed March 27, 2017.

47. Morschhauser F, Salles G, Le Gouill S, et al. A Phase IB study of obinutuzumab combined with lenalidomide for relapsed/refractory follicular B-cell lymphoma. Blood. 2014;124(21):4458.

48. Lymphoma Academic Research Organisation. Study of obinutuzumab combined to lenalidomide for the treatment of relapsed/refractory follicular and aggressive B-cell lymphoma. Available from: https://clinicaltrials.gov/ct2/show/NCT01582776. NLM identifier: NCT01582776. Accessed March 27, 2017.

49. Fowler N, Pinto RM, Cheah CY, et al. A phase I study of lenalidomide plus a next generation anti-CD20 antibody, obinutuzumab, in relapsed indolent lymphoma. Blood. 2015;126(23):2742.

50. MD Anderson Cancer Center. Study of obinutuzumab and lenalidomide in previously untreated subjects with follicular lymphoma. Available from: https://clinicaltrials.gov/ct2/show/NCT02871219. NLM identifier: NCT02871219. Accessed March 27, 2017.

51. Casulo C, Sancho JM, Van Eygen K, et al. Contempo: preliminary results in first-line treatment of follicular lymphoma with the oral dual PI3K- $\delta, \gamma$ inhibitor, duvelisib, in combination with rituximab or obinutuzumab. Blood. 2016;128(22):2979.

52. University of Munich. Combination of PCI-32765 with obinutuzumab in untreated follicular lymphoma. Available from: https:/clinicaltrials.gov/ ct2/show/NCT02689869. NLM identifier: NCT02689869. Accessed March 27, 2017.

53. Wahlin BE, Aggarwal M, Montes-Moreno S, et al. A unifying microenvironment model in follicular lymphoma: outcome is predicted by programmed death-1-positive, regulatory, cytotoxic, and helper T cells and macrophages. Clin Cancer Res. 2010;16(2):637-650.

54. Yang ZZ, Grote DM, Ziesmer SC, Xiu B, Novak AJ, Ansell SM. PD-1 expression defines two distinct T-cell sub-populations in follicular lymphoma that differentially impact patient survival. Blood Cancer J. 2015;5:e281.

55. Myklebust JH, Irish JM, Brody J, et al. High PD-1 expression and suppressed cytokine signaling distinguish $\mathrm{T}$ cells infiltrating follicular lymphoma tumors from peripheral T cells. Blood. 2013;121(8): 1367-1376.

56. Westin JR, Chu F, Zhang M, et al. Safety and activity of PD1 blockade by pidilizumab in combination with rituximab in patients with relapsed follicular lymphoma: a single group, open-label, phase 2 trial. Lancet Oncol. 2014;15(1):69-77.

57. Till BG, Park SI, Popplewell LL, et al. Safety and clinical activity of atezolizumab (anti-PDL1) in combination with obinutuzumab in patients with relapsed or refractory non-Hodgkin lymphoma. Blood. 2015;126(23):5104.

58. Davids MS, Roberts AW, Seymour JF, et al. Phase I first-in-human study of venetoclax in patients with relapsed or refractory non-Hodgkin lymphoma. J Clin Oncol. 2017;35(8):826-837.

59. Zelenetz AD, Salles GA, Mason KD, et al. Results of a phase IB study of venetoclax plus R- or G-CHOP in patients with B-cell non-Hodgkin lymphoma. Blood. 2016;128(22):3032. 
60. Swiss Group for Clinical Cancer Research. Obinutuzumab in combination with venetoclax in previously untreated follicular lymphoma patients. Available from: https:/clinicaltrials.gov/ct2/show/NCT02877550. NLM identifier: NCT02877550. Accessed March 27, 2017.

61. Ohio State University Comprehensive Cancer Center. Obinutuzumab, venetoclax, and lenalidomide in treating patients with relapsed or refractory B-cell non-Hodgkin lymphoma. Available from: https://clinicaltrials.gov/ct2/show/NCT02992522. NLM identifier: NCT02992522. Accessed March 27, 2017.

62. Phillips T, Brunvand M, Chen A, et al. Polatuzumab vedotin combined with obinutuzumab for patients with relapsed or refractory non-Hodgkin lymphoma: preliminary safety and clinical activity of a phase IB/II study. Blood. 2016;128(22):622.

63. Herrera AF, Matasar MJ, Assouline S, et al. Polatuzumab vedotin combined with bendamustine (B) and rituximab (R) or obinutuzumab (G) in patients with relapsed or refractory $(\mathrm{R} / \mathrm{R})$ follicular lymphoma (FL) or diffuse large B-cell lymphoma (DLBCL): preliminary results of a phase IB/II dose-escalation study. Blood. 2016;128(22):4194.

64. Hoffmann-La Roche. A study of obinutuzumab, polatuzumab vedotin, and venetoclax in relapsed or refractory follicular lymphoma (FL) or diffuse large B-cell lymphoma (DLBCL). Available from: https://clinicaltrials.gov/ct2/show/NCT02611323. NLM identifier: NCT02611323. Accessed March 27, 2017.

65. Gibiansky E, Gibiansky L, Carlile DJ, Jamois C, Buchheit V, Frey N. Population pharmacokinetics of obinutuzumab (GA101) in chronic lymphocytic leukemia (CLL) and non-Hodgkin's lymphoma and exposureresponse in CLL. CPT Pharmacometrics Syst Pharmacol. 2014;3:e144.
66. Gazyva ${ }^{\circledR}$ (obinutuzumab) [package insert]. San Francisco: Genentech; 2013 (revised in 2016).

67. Freeman CL, Morschhauser F, Sehn L, et al. Cytokine release in patients with CLL treated with obinutuzumab and possible relationship with infusion-related reactions. Blood. 2015;126(24):2646-2649.

68. van der Kolk LE, Grillo-Lopez AJ, Baars JW, Hack CE, van Oers MH. Complement activation plays a key role in the side-effects of rituximab treatment. Br J Haematol. 2001;115(4):807-811.

69. Byrd JC, Murphy T, Howard RS, et al. Rituximab using a thrice weekly dosing schedule in B-cell chronic lymphocytic leukemia and small lymphocytic lymphoma demonstrates clinical activity and acceptable toxicity. J Clin Oncol. 2001;19(8):2153-2164.

70. Byrd JC, Waselenko JK, Maneatis TJ, et al. Rituximab therapy in hematologic malignancy patients with circulating blood tumor cells association with increased infusion-related side effects and rapid blood tumor clearance. J Clin Oncol. 1999;17(3):791-795.

71. Winkler U, Jensen M, Manzke O, Schulz H, Diehl V, Engert A. Cytokine-release syndrome in patients with B-cell chronic lymphocytic leukemia and high lymphocyte counts after treatment with an anti-CD20 monoclonal antibody (rituximab, IDEC-C2B8). Blood. 1999;94(7): 2217-2224.

72. Dunleavy K, Tay K, Wilson WH. Rituximab-associated neutropenia. Semin Hematol. 2010;47(2):180-186.

73. Trotman J, Luminari S, Boussetta S, et al. Prognostic value of PET-CT after first-line therapy in patients with follicular lymphoma: a pooled analysis of central scan review in three multicentre studies. Lancet Haematol. 2014;1(1):e17-e27.
Cancer Management and Research

\section{Publish your work in this journal}

Cancer Management and Research is an international, peer-reviewed open access journal focusing on cancer research and the optimal use of preventative and integrated treatment interventions to achieve improved outcomes, enhanced survival and quality of life for the cancer patient The manuscript management system is completely online and includes

\section{Dovepress}

a very quick and fair peer-review system, which is all easy to use. Visit http://www.dovepress.com/testimonials.php to read real quotes from published authors. 